\title{
BREAST INFECTION
}

\author{
J M Dixon
}

\section{Organisms responsible for breast infections}

Type of breast infection

Organism

Neonatal

Lactating

Staphylococcus aureus

(Escherichia coli)*

Staphylococcus aureus

(Staphylococcus

epidermidis)*

(Streptococci)*

Non-lactating

Staphylococcus aureus

Enterococci

Anaerobic streptococci

Bacteroides spp

Skin associated Staphylococcus aureus

(Fungi)*

*Organisms only occasionally responsible.

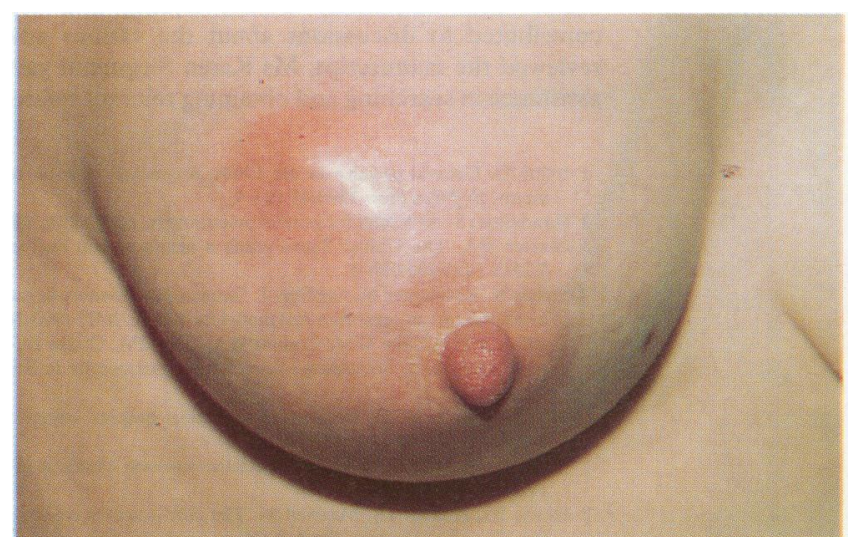

A breast abscess in right hand breast.

Breast infection is now much less common than it used to be. It is seen occasionally in neonates, but it most commonly affects women aged between 18 and 50; in this age group it can be divided into lactational and non-lactational infection. The infection can affect the skin overlying the breast, when it can be a primary event, or it may occur secondary to a lesion in the skin such as a sebaceous cyst or to an underlying condition such as hidradenitis suppurativa.

\section{Treatment} \section{infections* \\ Type of breast infection \\ Neonatal, lactating, and skin associated Non-lactating

$$
\begin{aligned}
& \text { penicillin } \\
& \text { Flucloxacillin } \\
& \text { (500 mg four } \\
& \text { times daily) } \\
& \text { Co-amoxiclav } \\
& \text { (375 mg thrice }
\end{aligned}
$$ \\ No allergy to \\ Flucloxacillin Co-amoxiclav daily)}

Antibiotics most appropriate for treating breast

*Doses are for adults.

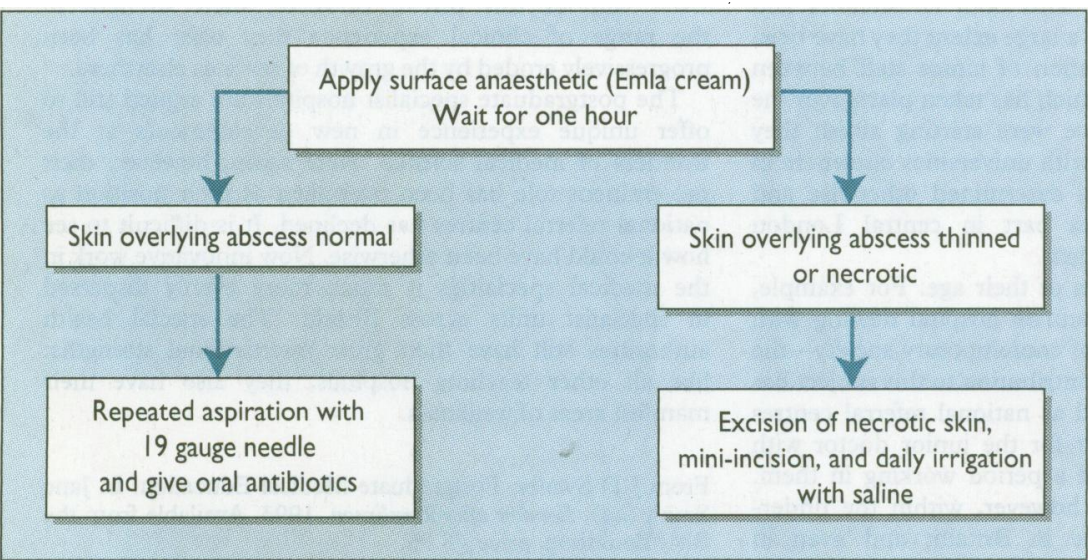

Protocol for treating breast abscesses.

\section{Allergy to penicillin}

Erythromycin $(500$ mg twice daily) $\mathrm{mg}$ four times daily) or erythromycin $(500 \mathrm{mg}$ twice daily) with metronidazole (200 mg thrice daily)
Combination of cephradine $(500$ by repeated aspiration or incision and drainage. Few breast abscesses require drainage under general anaesthesia except those in children, and placement of a drain after incision and drainage is unnecessary.
There are four guiding principles in treating breast infection

Appropriate antibiotics should be given does not settle rapidly with antibiotics - If an abscess is suspected it should be confirmed by aspiration before it is drained surgically

- Breast cancer should be excluded in patients with an inflammatory lesion which is despite apparently adequate treatment

All abscesses in the breast can be managed 


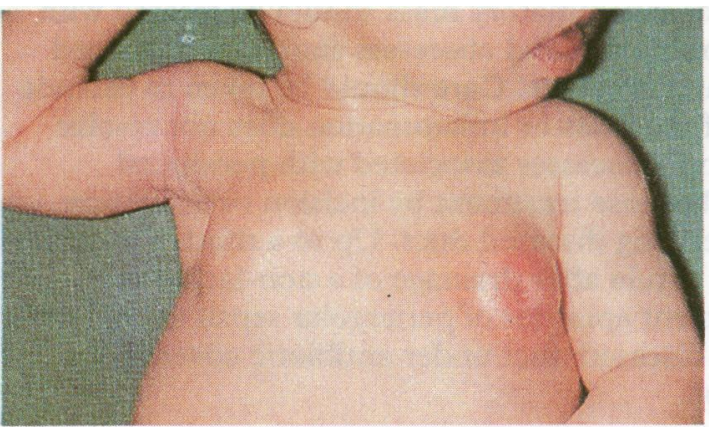

Neonatal breast abscess.

\section{Lactating infection}

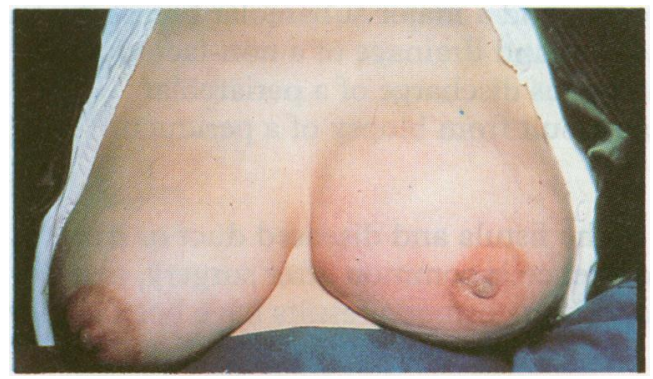

Puerperal mastitis of left breast. Note erythema, oedema, and obvious signs of inflammation, especially medially.

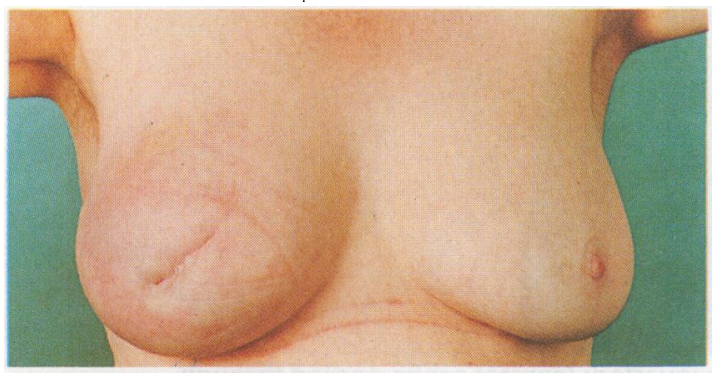

Inflammatory carcinoma of right breast. Note erythema and peau d'orange.

\section{Non-lactating infection}

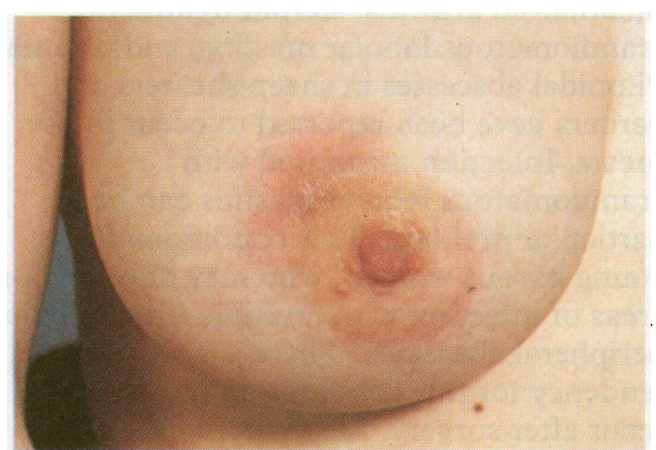

Periareolar inflammation due to periductal mastitis. Minor degree of nipple retraction at site of diseased duct.
Neonatal breast infection is most common in the first few weeks of life when the breast bud is enlarged. Although Staphylococcus aureus is the usual organism, Escherichia coli is occasionally the pathogen. If an abscess develops the incision to drain the pus should be placed as peripheral as possible to avoid damaging the breast bud.
Better maternal and infant hygiene and early treatment with antibiotics have considerably reduced the incidence of abscess formation during lactation. Infection is most frequently seen within the first six weeks of breast feeding, although some women develop it with weaning. Lactating infection presents with pain, swelling, and tenderness. There is usually a history of a cracked nipple or skin abrasion. Staphylococcus aureus is the most common organism responsible, but $S$ epidermidis and streptococci are occasionally isolated. Drainage of milk from the affected segment should be encouraged and is best achieved by continuing breast feeding. Tetracycline, ciprofloxacin, and chloramphenicol should not be used to treat lactating breast infection as they may enter breast milk and can harm the baby.

If the infection does not settle after a course of flucloxacillin and no pus is obtained on aspiration but the cytology indicates the lesion is infective or inflammatory, the antibiotic should be changed to coamoxiclav to cover other possible pathogens. If the inflammation or an associated mass lesion still persists then further investigations are required to exclude an underlying carcinoma. An established abscess should be treated by either recurrent aspiration or incision and drainage. Many women wish to continue to breast feed, and they should be encouraged to do so.

Non-lactating infections can be separated into those occurring centrally in the periareolar region and those affecting the peripheral breast tissue.

\section{Periareolar infection}

Periareolar infection is most commonly seen in young women with a mean age of 32. Histologically, there is active inflammation around nondilated subareolar breast ducts-a condition termed periductal mastitis. This condition has been confused with and called duct ectasia, but duct ectasia is almost certainly a separate condition affecting an older age group and characterised by subareolar duct dilatation and less pronounced and less active periductal inflammation. Current evidence suggests that smoking is an important factor in the aetiology of periductal mastitis but not in duct ectasia: about $90 \%$ of women who get periductal mastitis or its complications smoke cigarettes compared with $38 \%$ of the same age group in the general population. Substances in cigarette smoke may either directly or indirectly damage the wall of the subareolar breast ducts. The damaged tissues then become infected by either aerobic or anaerobic organisms. Initial presentation may be with periareolar inflammation (with or without an associated mass) or with an established abscess. Associated features include central breast pain, nipple retraction at the site of the diseased duct, and nipple discharge. 


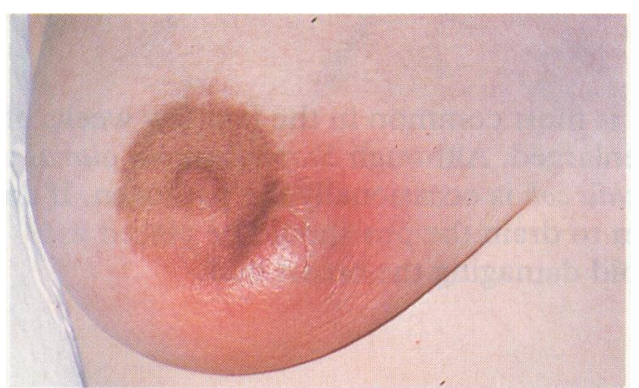

Non-lactating breast abscess due to periductal mastitis. Nipple retracted towards site of infection at areolar margin.

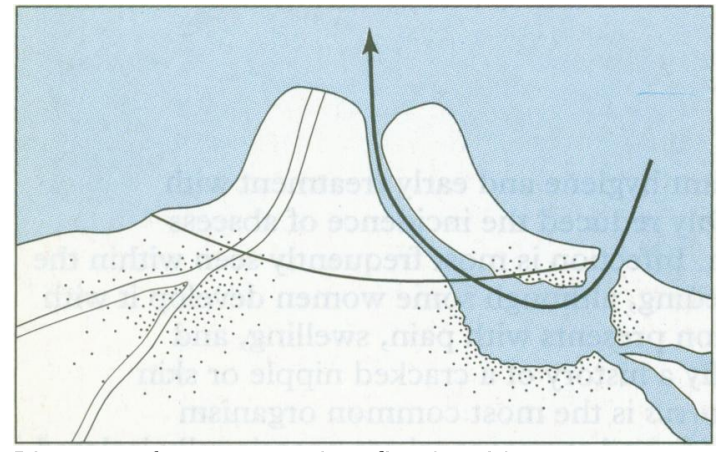

Diagram of mammary duct fistula with arrow showing path of fistula probe. Dots around left hand duct represent periductal mastitis, a precursor of a fistula.
Treatment - A periareolar inflammatory mass should be treated with a course of appropriate antibiotics, and abscesses should be managed by aspiration or incision and drainage. Care should be taken to exclude an underlying neoplasm if the mass or inflammation does not resolve after appropriate treatment. Abscesses associated with periductal mastitis commonly recur because treatment by incision or aspiration does not remove the underlying diseased duct. Up to a third of patients develop a mammary duct fistula after drainage of a non-lactating periareolar abscess. Recurrent episodes of periareolar sepsis should be treated by excision of the diseased duct under antibiotic cover by an experienced breast surgeon.

\section{Mammary duct fistula}

A mammary duct fistula is a communication between the skin usually in the periareolar region and a major subareolar breast duct. A fistula can develop after incision and drainage of a non-lactating abscess, it can follow spontaneous discharge of a periareolar inflammatory mass, or it can result from biopsy of a periductal inflammatory mass.

Treatment is by excision of the fistula and diseased duct or ducts under antibiotic cover. Recurrence is common after surgery, and the lowest rates of recurrence and best cosmetic results have been achieved in specialist breast units.
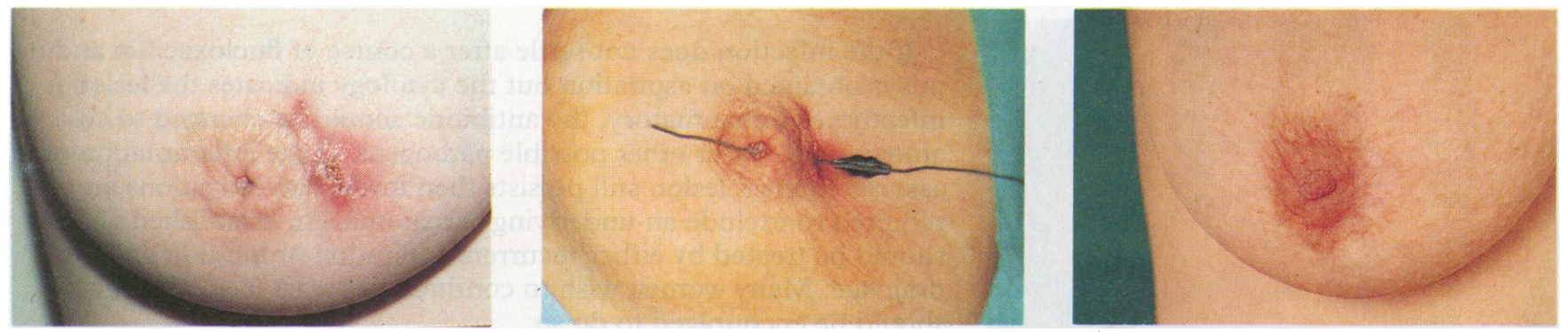

Mammary duct fistula: (left) external opening at areolar margin and whole of nipple inverted; (centre) probe passed through opening of fistula and emerging from affected duct; and (right) after excision of fistula and affected duct and primary wound closure under antibiotic cover. Operation performed through a circumareolar incision, which gives excellent cosmetic results.

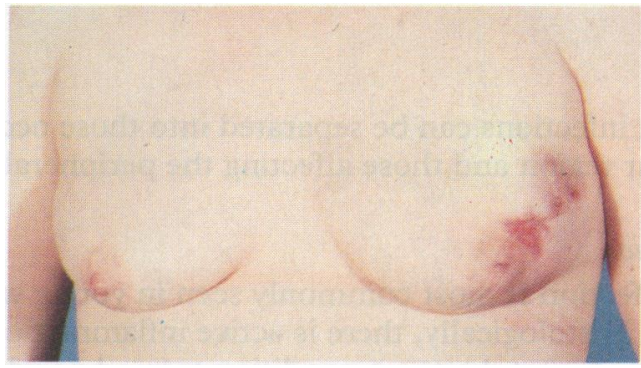

Granulomatous lobular mastitis of left breast. Multiple scars and nipple retraction.
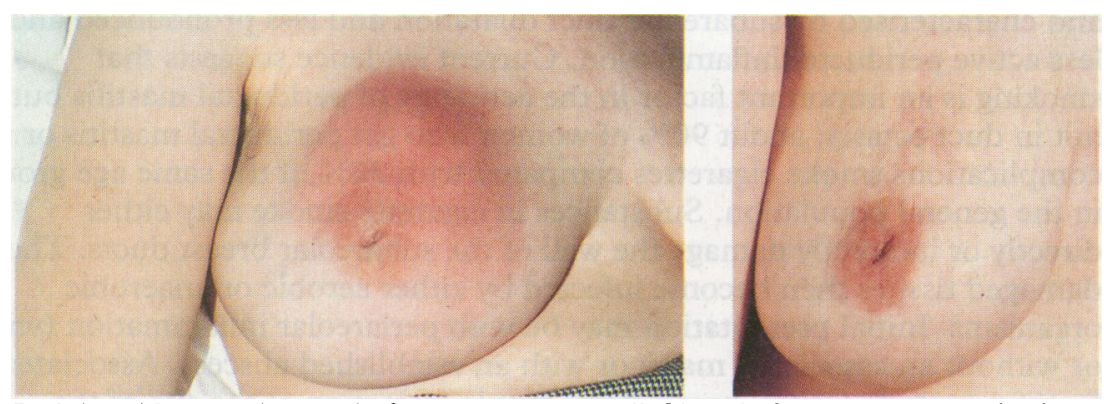

Peripheral breast abscess before management (left) and after recurrent aspiration and oral antibiotics (right).

\section{Peripheral non-lactating breast abscesses}

These are less common than periareolar abscesses and are often associated with an underlying condition such as diabetes, rheumatoid arthritis, steroid treatment, granulomatous lobular mastitis, and trauma. Pilonidal abscesses in sheep shearers and barbers have been reported to occur in the breast. Infection associated with granulomatous lobular mastitis can be a particular problem. This condition affects young parous women, who may develop large areas of infection with multiple simultaneous peripheral abscesses. There is a strong tendency for this condition to persist and recur after surgery. Large incisions and extensive surgery should therefore be avoided in this condition. Steroids have been tried but with limited success. Peripheral breast abscesses should be treated by recurrent aspiration or incision and drainage. 


\section{Skin associated infection}

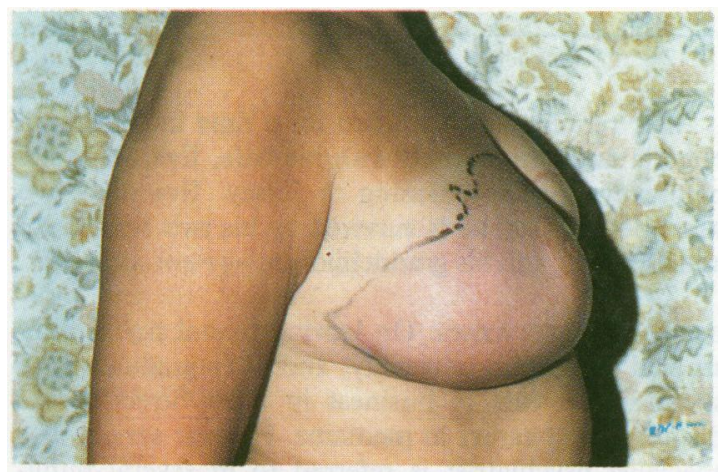

Cellulitis of right breast that occurred three years after uneventful reduction mammoplasty.

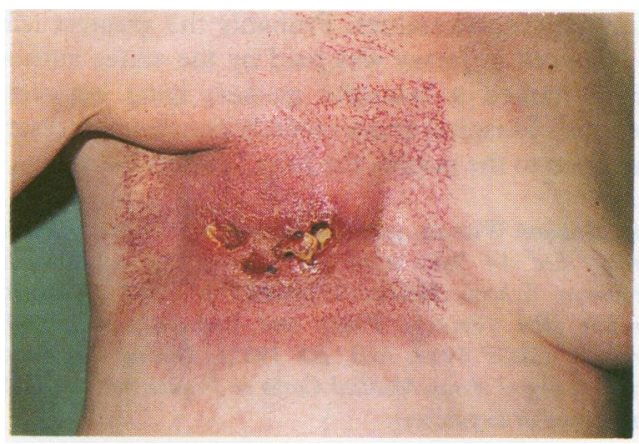

Cellulitis of right breast 10 years after radiotherapy.
Primary infection of the skin of the breast, which can present as cellulitis or an abscess, most commonly affects the skin of the lower half of the breast. These infections are often recurrent in women who are overweight, have large breasts, or have poor personal hygiene. Cellulitis most commonly affects the skin of the breast after surgery or radiotherapy. Staphylococcus aureus is the usual causative organism, although fungal infections have been reported.

Treatment of acute bacterial infection is with antibiotics and drainage or aspiration of abscesses. Women with recurrent infections should be advised about weight reduction and keeping the area as clean and dry as possible (this includes careful washing of the area up to twice a day, avoiding skin creams and talcum powder, and wearing either a cotton bra or a cotton $\mathrm{T}$ shirt or vest worn inside the bra.

Sebaceous cysts are common in the skin of the breast and may become infected. Some recurrent infections in the inframammary fold are due to hidradenitis suppurativa. In this condition the infection should first be controlled by a combination of appropriate antibiotics and drainage of any pus (the same organisms are found in hidradenitis as in non-lactating infection). Conservative excision of the affected skin is effective at stopping further infection in about half of patients; the remainder have further episodes of infection despite surgery.

\section{Other infections and conditions}

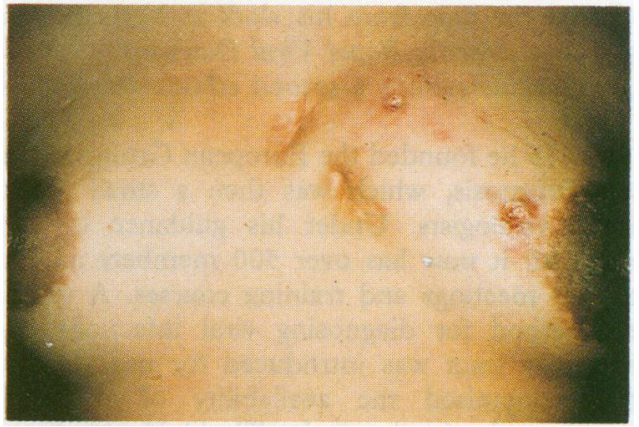

Tuberculosis of left breast with multiple sinuses.
Tuberculosis of the breast is now rare and can be primary or, more commonly, secondary. Clues to its diagnosis include the presence of a breast or axillary sinus in up to half of patients. The commonest presentation of tuberculosis nowadays is with an abscess resulting from infection of a tuberculous cavity by an acute pyogenic organism such as Staphylococcus aureus. An open biopsy is often required to establish the diagnosis. Treatment is by a combination of surgery and antituberculous chemotherapy.

Syphilis, actinomycosis, and mycotic, helminthic, and viral infections occasionally affect the breast but are rare.

\section{Factitial disease}

Artefactual or factitial diseases are created by the patient, often through complicated or repetitive actions. Such patients may undergo many investigations and operations before the nature of the disease is recognised. The diagnosis is difficult to establish but should be considered when the clinical situation does not conform to common appearances or pathological processes.
J M Dixon acknowledges the support of the Cancer Research Campaign. Photographs were prepared by Mr D Dirom, Medical Illustration Unit, University of Edinburgh

$\mathrm{J} M$ Dixon is senior lecturer in surgery at Edinburgh Royal Infirmary and honorary consultant surgeon at Western General Hospital, Edinburgh. 